EPJ Web of Conferences 106, 04001 (2016)

DOI: $10.1051 /$ epjconf/201610604001

(C) Owned by the authors, published by EDP Sciences, 2016

\title{
Use of Neutron Benchmark Fields for the Validation of Dosimetry Cross Sections
}

\author{
Patrick Griffin ${ }^{\mathrm{a}}$ \\ Sandia National Laboratories ${ }^{\mathrm{b}}$, Radiation Effects Sciences \& Applications Department, Albuquerque, \\ NM 87185-1146, USA
}

\begin{abstract}
The evolution of validation metrics for dosimetry cross sections in neutron benchmark fields is explored. The strength of some of the metrics in providing validation evidence is examined by applying them to the ${ }^{252} \mathrm{Cf}$ spontaneous fission standard neutron benchmark field, the ${ }^{235} \mathrm{U}$ thermal neutron fission reference benchmark field, the ACRR pool-type reactor central cavity reference benchmark fields, and the SPR-III fast burst reactor central cavity. The IRDFF dosimetry cross section library is used in the validation study and observations are made on the amount of coverage provided to the library contents by validation data available in these benchmark fields.
\end{abstract}

\section{Introduction}

The IRDFF dosimetry cross section library [1] includes more reactions than the previous IRDF-2002 library and extends the applicable energy range for incident neutrons up to $60 \mathrm{MeV}$. As this library is released to the public, it is important that the scope and extent of the validation of these cross sections be documented and available to the user community. When doing validation, it is important to note that any validation data must be accompanied by a quantitative statement on the uncertainty in the measured and the calculated quantity. Since differential cross section data, e.g. monoenergetic neutron cross section measurements were considered in the preparation of the evaluated cross sections, we look for validation, primarily, to comparisons with integral measurements obtained in benchmark neutron fields. The purpose of this paper is to explore various validation metrics and to compare the strength of the validation evidence that they can provide when applied to existing data in neutron benchmark fields.

\section{Validation Metrics}

The following sections address the evolution of various metrics that can serve to support validation arguments for cross section libraries and the application of these metrics to the validation of the

\footnotetext{
${ }^{\text {a }}$ Corresponding author: pjgriff@sandia.gov

${ }^{\mathrm{b}}$ Sandia is a multiprogram laboratory operated by Sandia Corporation, a Lockheed Martin Company, for the United States Department of Energy under contract DE-AC04-94AL85000.
}

This is an Open Access article distributed under the terms of the Creative Commons Attribution License 2.0, which permits unrestricted use, distribution, and reproduction in any medium, provided the original work is properly cited. 

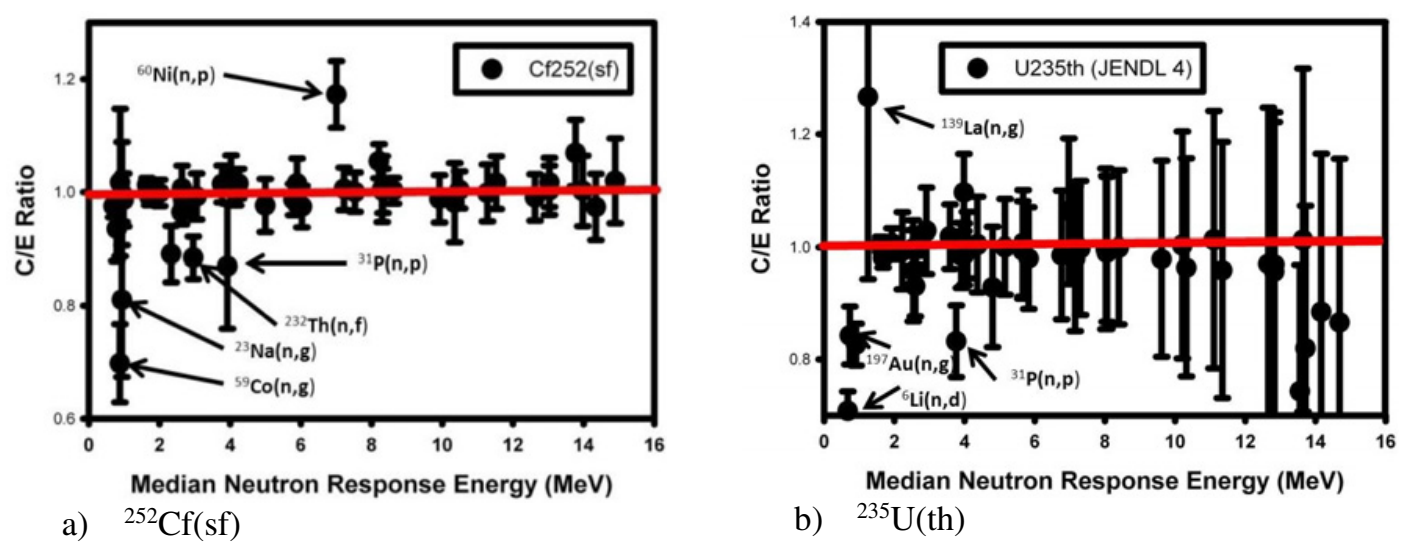

Figure 1. $\mathrm{C} / \mathrm{E}$ for Spectrum-averaged Cross Sections in the ${ }^{252} \mathrm{Cf}$ Spontaneous Fission Standard and ${ }^{235} \mathrm{U}$ Thermal Fission Reference Benchmark Neutron Field. Uncertainty includes spectra, cross section, and measurement terms.

IRDFF v.1.02 ${ }^{\mathrm{c}}$ standardized evaluated dosimetry cross section library [1]. The IRDFF library contains data for 76 reactions important to the dosimetry community. Observations will also be made on the scope of coverage provided by the validation evidence obtained for this set of reactions when the metrics are applied to datasets available from several benchmark fields.

\subsection{Spectrum-averaged Cross Sections}

Activity measurements for well characterized dosimetry reactions are the most common type of integral measurement taken in neutron fields. To take into account the irradiation conditions, these measurements are typically reported as spectrum-averaged cross sections in the neutron field. For well-characterized neutron fields, the neutron metrology community can obtain validation data by looking at a direct comparison of the calculated-to-experimental $(\mathrm{C} / \mathrm{E})$ ratios of the spectrum-averaged cross sections. Figure 1 shows the $\mathrm{C} / \mathrm{E}$ ratios for the cross section measurements in two benchmark neutron fields [1]. Figure 1a shows the $\mathrm{C} / \mathrm{E}$ ratios for the ${ }^{252} \mathrm{Cf}$ spontaneous fission field. Figure $1 \mathrm{~b}$ shows the data for the ${ }^{235} \mathrm{U}$ thermal fission field. There are many reported cross section measurements for some reactions in these benchmark fields. In our analysis we first made an a priori selection of the "best" cross section measurement, based on the reported uncertainty or the date of the measurement, and then computed the $\mathrm{C} / \mathrm{E}$ ratio. Multiple data-points for a single reaction are not displayed in this figure because the same dataset was subsequently used to support the analysis with other validation metrics. In some of the other analysis, the use of multiple data points for a single reaction would have biased the analysis process, e.g. the least squares analysis as reported in Sect. 2.3 (unless one was able to quantify the correlation between the multiple data points for the same reaction).

The ${ }^{252} \mathrm{Cf}$ spontaneous fission standard neutron benchmark field shows excellent agreement between the measured and calculated spectrum-averaged cross sections for most reactions. Exceptions include the ${ }^{60} \mathrm{Ni}(\mathrm{n}, \mathrm{p}),{ }^{31} \mathrm{P}(\mathrm{n}, \mathrm{p})$, and ${ }^{232} \mathrm{Th}(\mathrm{n}, \mathrm{f})$ reactions. In addition, there are issues with low energy $(\mathrm{n}, \gamma)$ reactions - but these differences for reactions dominated by the low energy neutron response that may be attributable to incorrect measurement corrections applied for room scatter in the neutron field.

The Fig. 1b data for the ${ }^{235} \mathrm{U}$ thermal fission field also shows good agreement, but show much larger uncertainties. These uncertainties are driven by the spectral uncertainty component. When one

${ }^{\mathrm{c}}$ IRDFF Version 1.03 became available on March 4, 2014, but this paper addresses version 1.02. 
looks at calculated-to-experimental ratios for the spectrum-averaged cross section in this less well characterized ${ }^{235} \mathrm{U}$ thermal fission spectrum, one can see a systematic energy-dependent trend in the $\mathrm{C} / \mathrm{E}$ spectrum-averaged cross section ratios [2]. This trend can lead one to postulate a systematic problem with the spectrum, but the large uncertainty bands prevent one from recommending any action to resolve the discrepancy in the underlying nuclear data, i.e. the cross section or spectrum, measurements. Note that the issue here addresses the spectrum as exhibited in the macroscopic benchmark field and conclusions drawn here should not necessarily be attributed to the microscopic ${ }^{235} \mathrm{U}$ thermal fission cross section [3]. At low energy, the ${ }^{139} \mathrm{La}(\mathrm{n}, \gamma)$ reaction, with a median energy response of 1.2495 MeV, shows a significant deviation from unity, but it also has a very large experimental measurement uncertainty and is still seen to be within 1 -sigma. In the mid-energy region, the ${ }^{31} \mathrm{P}(\mathrm{n}, \mathrm{p})$ reaction is seen to be discrepant. The other three reactions with a low energy median response energy $\left[{ }^{6} \operatorname{Li}(\mathrm{n}, \mathrm{d}),{ }^{10} \mathrm{~B}(\mathrm{n}, \alpha)\right.$, and $\left.{ }^{197} \mathrm{Au}(\mathrm{n}, \gamma)\right]$ have a much smaller overall uncertainty but show significant deviations from unity. These measurements come from the NBS measurements [4]. The ${ }^{10} \mathrm{~B}(\mathrm{n}, \alpha)$ and ${ }^{6} \mathrm{Li}(\mathrm{n}, \mathrm{d})$ reactions are discussed in more detail in Sect. 2.2.2.

\subsection{Spectral Indices}

Historically, when comparisons with integral benchmarks have been made, rather than reporting a spectrum-averaged cross section, the analyst has used, as the metric of interest, a spectral index (SI) [4]. A spectral index is the ratio of the spectral-averaged cross section for two different reactions, a detector and a reference reaction, gathered in a neutron field for the same exact irradiation. This metric is often used as an experimental metric because it eliminates the uncertainty due to the knowledge of the intensity (fluence) of the neutron benchmark field during the irradiation. The value of any metric in a validation application comes from the ability to compare the experimental value to a computed value, i.e. a calculated-to-experimental $(\mathrm{C} / \mathrm{E})$ ratio. For neutron fields that lack a high fidelity uncertainty characterization of the neutron spectrum, the use of the spectral index as the validation metric has typically neglected to include this spectral uncertainty contributor [5]. This neglect can significantly reduce the reported value for the uncertainty in the calculated SI value. The spectrum uncertainty contribution is often larger than the cross section uncertainty contribution in an individual spectrumaveraged cross section. One advantage of reporting a spectral index rather than a spectrum-averaged cross section is that, for this metric, the contribution from the spectral uncertainty in the SI ratio can be significantly reduced, relative to that for the spectrum-averaged cross section, when the energydependent sensitivity of the cross sections appearing in both the numerator and denominator of the SI ratio span a similar energy range. A good example of the historical use of spectral indices for cross section validation can be found in Reference 4. The following subsections use the data found in Reference 4 and examine the consistency of the experimentally-derived spectral indices with the computed spectral indices that result from using the best available neutron spectrum and the IRDFF cross sections.

\subsubsection{Application to ${ }^{252}$ Cf Spontaneous Fission Standard Benchmark Neutron Field}

Figure 2 shows the C/E SI ratios, with an error bar representing the 1-sigma uncertainty level, for the spectral indices in this standard benchmark neutron field. The reference reaction for the SIs is the ${ }^{238} \mathrm{U}(\mathrm{n}, \mathrm{f})$ reaction. Figure 2a shows the original 13-point C/E data as published in the 1986 NBS report. Figure $2 b$ show the updated results when the IRDFF v.1.02 cross sections are used. In Fig. $2 b$ the C/E ratios were computed using the Mannhart ${ }^{252} \mathrm{Cf}$ spontaneous fission neutron spectrum [6], the Grundl measurements [4], and the IRDFF cross sections [1]. The discrepant outlier in Fig. 2a near $3.7 \mathrm{MeV}$ is the ${ }^{47} \mathrm{Ti}(\mathrm{n}, \mathrm{p})$ reaction. The cause of this discrepancy was previously discovered by Mannhart [7] and the modified cross section for this reaction was reflected in the earlier IRDF-2002 cross sections. But, in 


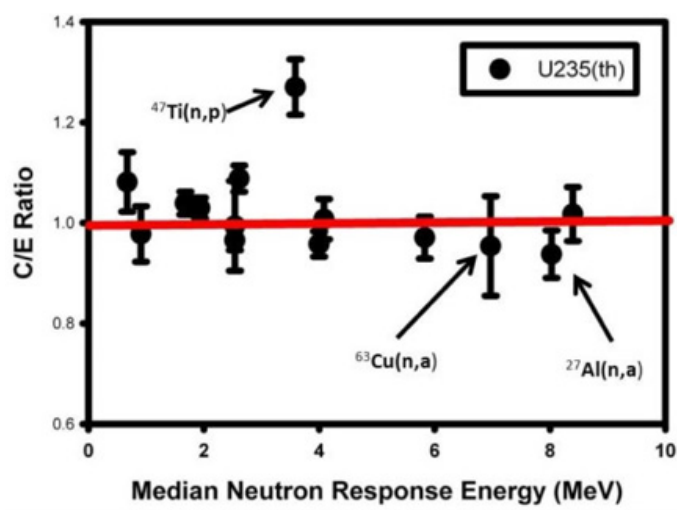

a) Original NBS reported SI C/E

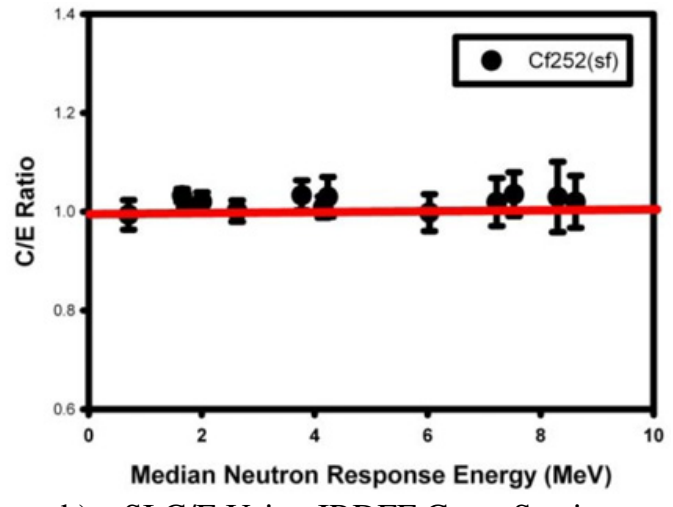

b) SI C/E Using IRDFF Cross Sections

Figure 2. C/E SI Ratios in the ${ }^{252} \mathrm{Cf}$ Spontaneous Fission Standard Benchmark Neutron Field.

addition to this change, one can see that many of the $\mathrm{C} / \mathrm{E}$ ratios have been improved, in particular the ${ }^{63} \mathrm{Cu}(\mathrm{n}, \alpha)$ reaction, with a median response energy at $7.22 \mathrm{MeV}$, and the ${ }^{27} \mathrm{Al}(\mathrm{n}, \alpha)$ reaction at $8.6 \mathrm{MeV}$ have improved $\mathrm{C} / \mathrm{E}$ values. A small systematic offset is seen for the low energy $\mathrm{C} / \mathrm{E}$ values (4 of the lowest 6 points in Fig. 2b), but all values remain within the 1-sigma interval.

Because the ${ }^{252} \mathrm{Cf}$ spontaneous fission source is the best characterized neutron benchmark field with a neutron spectrum characterized by time-of-flight measurements, the spectrum uncertainty is not a significant contributor to the overall uncertainty. The importance of the spectrum contribution to the uncertainty in the calculated SI in other less well characterized fields is discussed in reference [5].

\subsubsection{Application to ${ }^{235} \mathrm{U}$ Thermal Fission Reference Benchmark Neutron Field}

Figure 3 shows the C/E SI ratios for the spectral indices in this reference benchmark neutron field. The reference reaction for the SIs is the ${ }^{238} \mathrm{U}(\mathrm{n}, \mathrm{f})$ reaction. Figure 3a shows the original 14-point $\mathrm{C} / \mathrm{E}$ data as originally published in the 1986 NBS report. In this calculation the ${ }^{235} \mathrm{U}$ NBS spectrum is used along with the ENDF/B-V cross section. Figure $3 \mathrm{~b}$ show the updated results when the IRDFF cross sections are used. In Fig. $3 b$ the C/E ratios were computed using the JENDL $4.0{ }^{235} \mathrm{U}$ thermal fission neutron spectrum [8], the Grundl measurements [4], and the IRDFF cross sections [1]. The ${ }^{47} \mathrm{Ti}(\mathrm{n}, \mathrm{p})$ reaction discrepant $\mathrm{C} / \mathrm{E}$ [7] in the original data is again seen to be fixed with the latest cross sections. The JENDL spectrum has a larger uncertainty that the NBS spectrum, and this results in the larger error bars in the high energy reactions in Fig. $3 b$.

The low energy ${ }^{10} \mathrm{~B}(\mathrm{n}, \alpha)$ and ${ }^{6} \mathrm{Li}(\mathrm{n}, \mathrm{t})$ reactions are seen to be much more discrepant in Fig. $3 \mathrm{~b}$ than in Fig. 3a. The author first, erroneously, thought that this difference in validation results may be related to the use of a coarse group structure representation of the ${ }^{235} \mathrm{U}(\mathrm{th})$ spectrum in the original NBS reported analysis and to the presence of a low energy room-scatter neutron contribution to the measurement. However, work recently reported by Simakov [10] clearly indicates that the discrepancy seen in Fig. 3b is due to a failure to include other helium production reactions in this analysis. Reference 10 shows that when the ${ }^{10} \mathrm{~B}(\mathrm{n}, \mathrm{t} 2 \alpha)$ reaction is added into the analysis, the $\mathrm{C} / \mathrm{E}$ for the ${ }^{10} \mathrm{~B}(\mathrm{n}, \alpha)$ analysis is $\sim 1.0$. Similarly, when the ${ }^{6} \mathrm{Li}(\mathrm{n}, \mathrm{nd} \alpha)$ and ${ }^{6} \mathrm{Li}(\mathrm{n}, 2 \mathrm{np} \alpha)$ reactions are included in the analysis, the ${ }^{6} \mathrm{Li}(\mathrm{n}, \mathrm{t})^{4} \mathrm{He}$ reaction gives a $\mathrm{C} / \mathrm{E}$ of $\sim 1.03$. The original NBS measurements for ${ }^{10} \mathrm{~B}$ and ${ }^{6} \mathrm{Li}$ were labelled as "helium production" reactions and obviously addressed the composite reaction and thus should be treated as ${ }^{10} \mathrm{~B}(\mathrm{n}, \mathrm{X}){ }^{4} \mathrm{He}$ and ${ }^{6} \mathrm{Li}(\mathrm{n}, \mathrm{X}){ }^{4} \mathrm{He}$ reactions. 


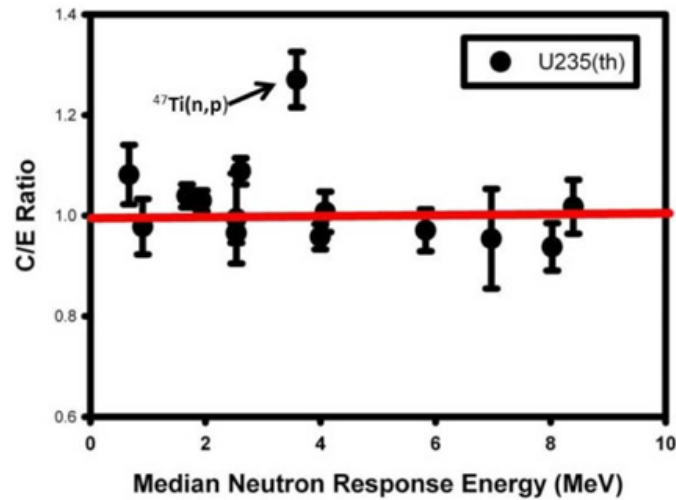

a) Original NBS reported SI C/E

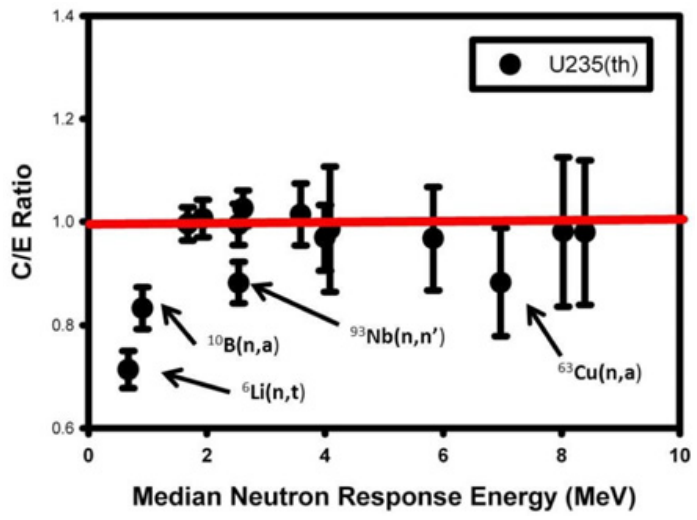

b) SI C/E Using IRDFF Cross Sections

Figure 3. C/E SI Ratios in the ${ }^{235} \mathrm{U}$ Thermal Fission Reference Benchmark Neutron Field.

The updated SI for the ${ }^{93} \mathrm{Nb}$ (n.n') reaction, with a median energy response of $2.54 \mathrm{MeV}$, is also seen to be more discrepant. This reaction also was not present in the NBS C/E analysis in the ${ }^{252} \mathrm{Cf}$ field, so again we cannot look there for validation evidence. The ${ }^{63} \mathrm{Cu}(\mathrm{n}, \alpha)$ reaction, with a median response energy of $6.97 \mathrm{MeV}$, has a $\mathrm{C} / \mathrm{E}$ that deviated from unity more than with the IRDFF cross sections, but is still (just) within 1-sigma. The uncertainty bands here are significantly larger than those seen for the ${ }^{252} \mathrm{Cf}$ standard field, but, except for the reactions mentioned above, still show good agreement. The uncertainty in the latest JENDL $4.0{ }^{235} \mathrm{U}$ fission spectrum is also much larger than that found in the NBS spectral representation, e.g. at $8 \mathrm{MeV}$ JENDL 4.0 has a ${ }^{235} \mathrm{U}$ thermal fission spectrum uncertainty of $13 \%$ while the NBS spectrum used an uncertainty of only $5.3 \%$.

\subsection{Combined Least Squares}

One approach that can isolate the consistency of the spectrum-averaged cross sections in a less well characterized neutron field, compensating for the a priori uncertainty in the knowledge of the neutron spectrum, is to do a least squares-based spectrum adjustment for the neutron field. This approach, when applied to the ${ }^{235} \mathrm{U}$ thermal fission spectrum, can be seen to significantly reduce the error bars and make it easier to see if there is a systematic trend in the reported C/E ratios [9]. A comparison of error bounds with that from the earlier validation metrics shows that it provides a better metric of the $\mathrm{C} / \mathrm{E}$ for the consistency of the underlying nuclear data. One critical caveat here is that one cannot use an a priori spectrum in this least squares adjustment that was derived through the use of spectrum-averaged cross sections unless this correlation between spectrum and cross sections is properly taken into account. Thus, one has to be very careful that an adjusted cross section obtained from a cross section validation activity is clearly labelled as such and never makes its way back into the literature as an unbiased spectrum representation.

\subsubsection{Application to ${ }^{252}$ Cf Spontaneous Fission Standard Benchmark Neutron Field}

The initial least square analysis of the ${ }^{252} \mathrm{Cf}$ field used 48 sensors/activation measurements and yielded an unacceptable $\chi^{2}$ degree of freedom (dof) of 2.65. Using the criteria of rejecting foils that had a C/E that exceeded 3-sigma, the analysis then removed the most discrepant reactions, ${ }^{59} \operatorname{Co}(\mathrm{n}, \gamma),{ }^{92} \mathrm{Mo}(\mathrm{n}, \mathrm{p})$, and ${ }^{232} \mathrm{Th}(\mathrm{n}, \mathrm{f})$, producing an acceptable spectrum with a $\chi^{2} /$ dof of 1.28 . 

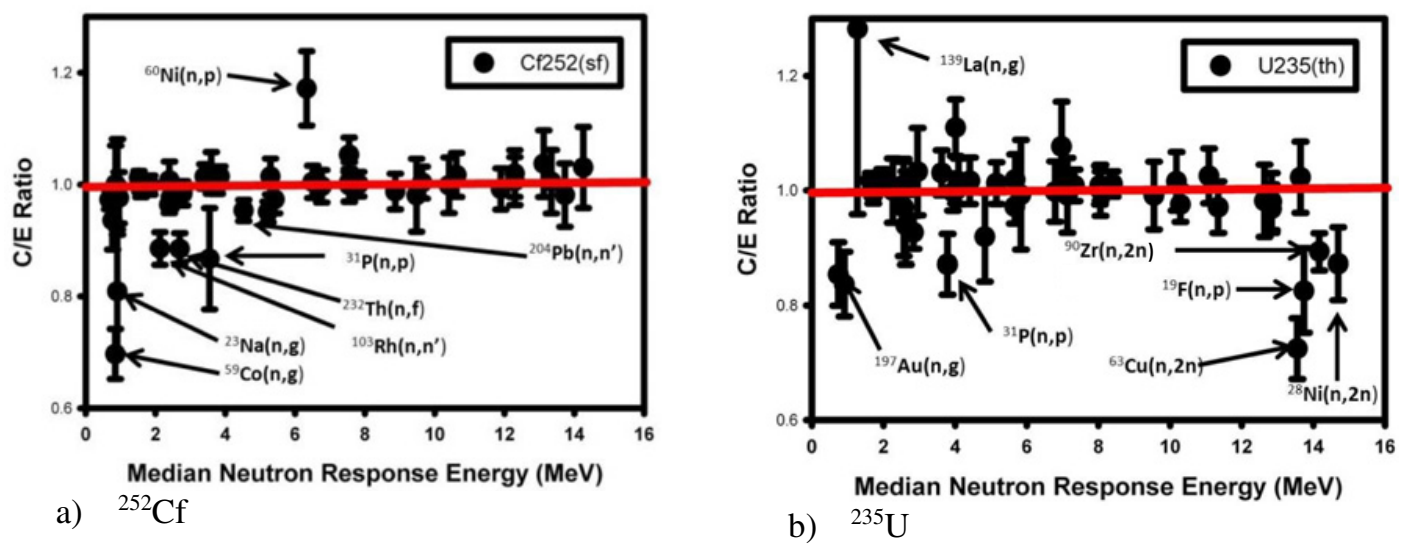

Figure 4. $\mathrm{C} / \mathrm{E}$ for Spectrum-averaged Cross Sections from Least Square Analysis of the ${ }^{252} \mathrm{Cf}$ and ${ }^{235} \mathrm{U}$ Benchmark Neutron Fields.

Figure 4a shows good agreement and small 1-sigma C/E values for the majority of the reactions, but discrepancies greater than 1-sigma are seen for:

- fast neutron response reactions: ${ }^{60} \mathrm{Ni}(\mathrm{n}, \mathrm{p}),{ }^{31} \mathrm{P}(\mathrm{n}, \mathrm{p}),{ }^{232} \mathrm{Th}(\mathrm{n}, \mathrm{f}),{ }^{103} \mathrm{Rh}\left(\mathrm{n}, \mathrm{n}^{\prime}\right),{ }^{204} \mathrm{~Pb}\left(\mathrm{n}, \mathrm{n}^{\prime}\right)$

- low-energy neutron response reactions: ${ }^{23} \mathrm{Na}(\mathrm{n}, \gamma),{ }^{59} \mathrm{Co}(\mathrm{n}, \gamma)$.

\subsubsection{Application to ${ }^{235} U$ Thermal Fission Reference Benchmark Neutron Field}

Figure $4 \mathrm{~b}$ shows the good agreement in the $\mathrm{C} / \mathrm{E}$ ratios in the ${ }^{235} \mathrm{U}(\mathrm{th})$ thermal fission reference benchmark field. The 1-sigma error bounds in these $\mathrm{C} / \mathrm{E}$ ratios are seen to be much smaller than that seen for the spectrum-averaged cross section metric in Fig. $1 \mathrm{~b}$.

Issues are seen for the ${ }^{235} \mathrm{U}\left(\right.$ th) field for the ${ }^{19} \mathrm{~F}(\mathrm{n}, 2 \mathrm{n}),{ }^{63} \mathrm{Cu}(\mathrm{n}, 2 \mathrm{n}),{ }^{90} \mathrm{Zr}(\mathrm{n}, 2 \mathrm{n})$, and ${ }^{58} \mathrm{Ni}(\mathrm{n}, 2 \mathrm{n})$ high energy response reactions. Note that the ${ }^{252} \mathrm{Cf}$ data provided good validation evidence for the last three of these cross sections. An inspection of validation data in the EXFOR database obtained in 14-MeV DT accelerator produced neutron fields shows good validation evidence for all four of these reactions - but it also shows significant variation in reported EXFOR measurements: $(\sim 50 \%)$ for the ${ }^{19} \mathrm{~F}(\mathrm{n}, 2 \mathrm{n})$ and ${ }^{58} \mathrm{Ni}(\mathrm{n}, 2 \mathrm{n})$ reactions and $\sim 20 \%$ for the ${ }^{63} \mathrm{Cu}(\mathrm{n}, 2 \mathrm{n})$ cross sections in this region. One might look for an issue with the ${ }^{235} \mathrm{U}$ spectrum, but the ${ }^{89} \mathrm{Y}(\mathrm{n}, 2 \mathrm{n}){ }^{235} \mathrm{U}($ th) activity is sensitive in the same energy region and appears to be in agreement. The path forward here is not clear, but the data clearly indicates discrepant data in the high energy ( $>13 \mathrm{MeV}$ ) region. Thus the discrepancy appears to lie with either the measurement data or the a priori JENDL-4.0 ${ }^{235} \mathrm{U}$ thermal fission neutron spectrum. The discrepancy could reside in an invalid/smaller uncertainty being assigned to the spectrum or measurement data.

We note that a new release of the IRDFF library, version 1.03, has just been made available and that this library changes the ${ }^{58} \mathrm{Ni}(\mathrm{n}, 2 \mathrm{n})$ cross section. However, initial work with the LSL spectrum unfold shows that use of this newer cross section evaluation does not solve the problem with the ${ }^{58} \mathrm{Ni}(\mathrm{n}, 2 \mathrm{n})$ reaction. It improves the agreement for the ${ }^{58} \mathrm{Ni}(\mathrm{n}, 2 \mathrm{n})$ reaction in the ${ }^{235} \mathrm{U}(\mathrm{th})$ field, but decreases the good agreement seen in the ${ }^{252} \mathrm{Cf}(\mathrm{sf})$ field.

\subsubsection{Application to ACRR Central Cavity Reference Benchmark Neutron Field}

The Annular Core Research Reactor (ACRR) at Sandia National Laboratories is a pool-type reactor configured as a reference field used for radiation damage studies on electronics. A least square analysis 


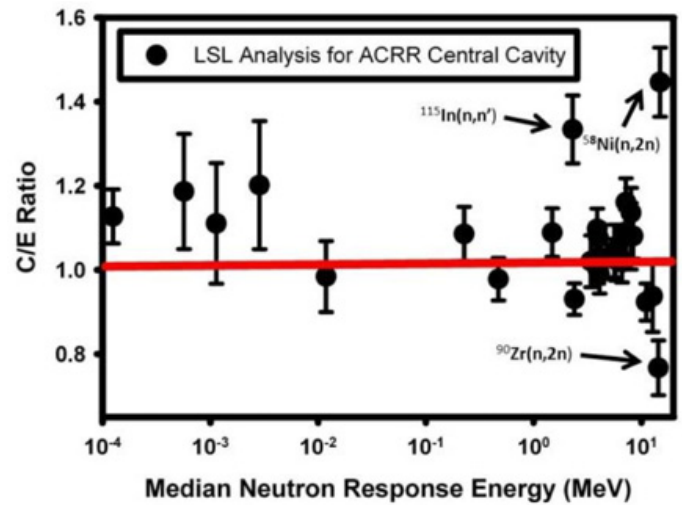

a) Logarithmic Energy

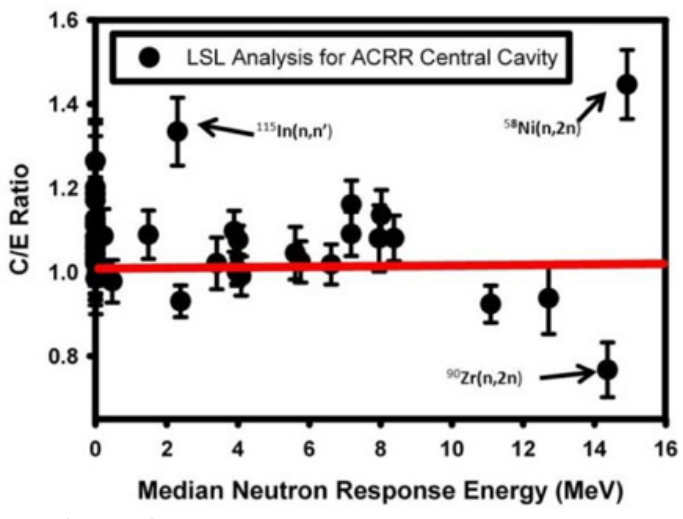

b) Linear Energy

Figure 5. C/E for Spectrum-averaged Cross Sections in the ACRR Central Cavity Reference Benchmark Neutron Field.

was performed on activation data gathered in this field using 40 foils resulting in a chi-squared peer degree of freedom of 2.06. Figure 5 shows the C/E ratios for the spectrum-averaged cross sections. Inserts to the figure depict both a linear and a logarithmic energy axis in order to increase the visibility of the consistency of the metrics. The error bars include uncertainty contributions from the activation measurement, the spectrum as determined by a least-squares analysis, and the IRDFF cross sections. Most of the 40 reactions for which activation data were available show good agreement, i.e. approximately within the 1-sigma level indicated by the error bars. Exceptions to this good agreement include:

- The ${ }^{58} \mathrm{Ni}(\mathrm{n}, 2 \mathrm{n})$ and ${ }^{90} \mathrm{Zr}(\mathrm{n}, 2 \mathrm{n})$ which are in conflict in the high energy region whereas they were discrepant but consistent for ${ }^{235} \mathrm{U}(\mathrm{th})$, as depicted in Fig. $4 \mathrm{~b}$.

- The ${ }^{115} \operatorname{In}(n, n$ ') reaction, for which some questions have been raised about the measurement.

\subsubsection{Application to SPR-III Central Cavity Reference Benchmark Neutron Field}

The Sandia Pulse Reactor (SPR-III) at Sandia National Laboratories was a fast burst reactor configured as a reference field used for radiation damage studies on electronics. A least square analysis was performed on activation data gathered in this field using 31 foils resulting in a chi-squared per dof of 2.193. Figure 6 shows the $\mathrm{C} / \mathrm{E}$ ratios for the spectrum-averaged cross sections. Inserts to the figure depict both a linear and a logarithmic energy axis to increase the visibility of the consistency of the metrics. Discrepant reactions were found for $(\mathrm{n}, \gamma)$ reactions on ${ }^{197} \mathrm{Au},{ }^{45} \mathrm{Sc}$, and ${ }^{55} \mathrm{Mn}$ reactions. New validation evidence was found for 5 reactions/covers covering 3 reactions.

\subsection{Status of Validation for IRDFF Library}

This analysis:

- examined measurements for 49 reactions in the ${ }^{252} \mathrm{Cf}(\mathrm{sf})$ standard benchmark field which provided validation evidence for 41 reactions;

- examined measurements for 47 reactions in the ${ }^{235} \mathrm{U}($ th) reference benchmark field which provided validation evidence for 42 reactions, 9 of which were not validated from the data in the ${ }^{252} \mathrm{Cf}(\mathrm{sf})$ field; 


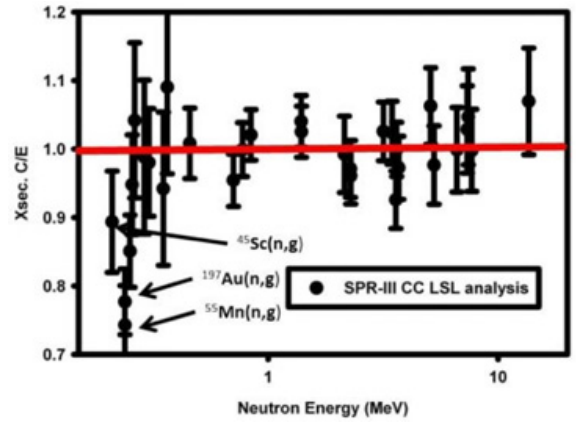

c) Logarithmic Energy

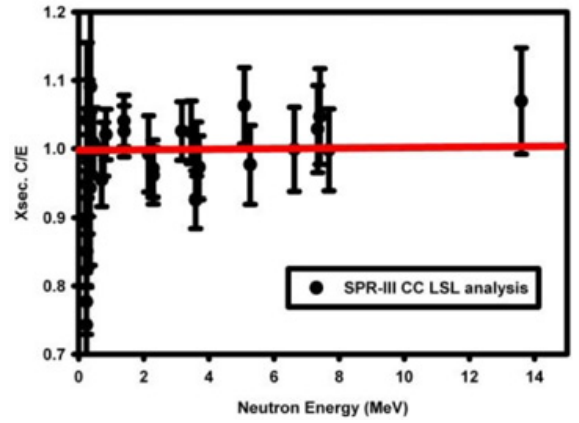

d) Linear Energy

Figure 6. $\mathrm{C} / \mathrm{E}$ for Spectrum-averaged Cross Sections in the SPR-III Central Cavity Reference Benchmark Neutron Field.

- examined measurements for 41 reaction/cover combinations on the ACRR central cavity reference benchmark field which provided validation evidence for 31 reactions, 5 of which were not validated by the data in either the ${ }^{252} \mathrm{Cf}(\mathrm{sf})$ or ${ }^{235} \mathrm{U}$ (th) fields.

- Examined measurements for 31 reaction/cover combinations in the SPR-III central cavity reference benchmark field which provided validation evidence for 27 reaction/covers, including 3 reactions which were not validated in other fields.

Thus this analysis reports on validation evidence for 58 of the 76 reactions in the IRDFF library. Concern is raised that the following cross sections are discrepant:

- ${ }^{31} \mathrm{P}(\mathrm{n}, \mathrm{p})$ which is discrepant in both the ${ }^{252} \mathrm{Cf}(\mathrm{sf})$ and ${ }^{235} \mathrm{U}(\mathrm{th})$ fields.

- ${ }^{55} \mathrm{Mn}(\mathrm{n}, \gamma)$ which is discrepant in the ACRR field.

Conflicting data between reactions measurements in some fields suggest that priority be given to repeating the following measurements:

- in the ${ }^{252} \mathrm{Cf}$ (sf) field: ${ }^{60} \mathrm{Ni}(\mathrm{n}, \mathrm{p}),{ }^{103} \mathrm{Rh}\left(\mathrm{n}, \mathrm{n}\right.$ '), ${ }^{204} \mathrm{~Pb}\left(\mathrm{n}, \mathrm{n}\right.$ '), ${ }^{232} \mathrm{Th}(\mathrm{n}, \mathrm{f})$ \{all reactions validated in the ${ }^{235} \mathrm{U}(\mathrm{th})$ field but discrepant here .

- in the ${ }^{235} \mathrm{U}\left(\right.$ th) field: ${ }^{63} \mathrm{Cu}(\mathrm{n}, 2 \mathrm{n}),{ }^{19} \mathrm{~F}(\mathrm{n}, 2 \mathrm{n}),{ }^{90} \mathrm{Zr}(\mathrm{n}, 2 \mathrm{n}),{ }^{58} \mathrm{Ni}(\mathrm{n}, 2 \mathrm{n})$ \{all reactions validated in the ${ }^{252} \mathrm{Cf}(\mathrm{sf})$ field but discrepant here $\}$.

Eighteen reactions in the IRDFF 1.02 library are still lacking in validation evidence. Many of these remaining reactions are not ones which lend themselves toward easily gathering such evidence in typical reactor neutron fields, for example they either have a very high threshold energy, a short half-life, or a low gamma yield. If these reactions have value to the dosimetry community; efforts should be focused on gathering relevant validation evidence for these reactions.

\section{Conclusions and Recommendations}

The usefulness of several validation metrics has been addressed and the value of the metrics demonstrated by applying them to the ${ }^{235} \mathrm{U}$ thermal fission, ${ }^{252} \mathrm{Cf}$ spontaneous fission, and to reactor reference fields for which there exists a good database of measured dosimetry cross sections. A coupled least squared analysis that includes the cross section along with the spectral representation and the measurements appears to be the most useful validation metric. 


$$
15^{\text {th }} \text { ISRD }
$$

\section{References}

[1] International Reactor Dosimetry and Fusion File, IRDFF v.1.02, June 14, 2012, see https: //www-nds.iaea.org/IRDFF/

[2] R. Capote et al., IAEA report INDC(NDS)-0608

[3] N. Kornilov, International Journal of Nuclear Energy Science and Engineering, Vol. 2 (2012)

[4] J.A. Grundl, C.M. Eisenhauer, NBSIR 85-03151, Compendium of Benchmark Neutron Fields for Reactor Dosimetry, US Department of Commerce, National Bureau of Standards, January 1986

[5] P. J. Griffin, Covariance Propagation in Spectral Indices, presentation at the International Workshop on Nuclear Data Covariances, April 28 - May 1, 2014 in Santa Fe, New Mexico. Accepted for publication in Nuclear Data Sheets, January 2015

[6] Cf-252 Neutron Spectrum, Ed. H.D. Lemmel, IAEA report, IAEA-NDS-98, Dec. 1987 (includes W. Mannhart reports)

[7] W. Mannhart, D.L. Smith, J.W. Meadows, "The Discrepancy Between Differential and Integral Data on the Ti-47(n,p)", in American Nuclear Society Proceedings on a Topical Meeting in Advances in Nuclear Engineering Computation and Radiation Shielding, Santa Fe, New Mexico, April 9-13, 1989, University of New Mexico Publications, Ed. M.L. Hall, pp. 577-580

[8] K. Shibata, et al., J. Katakura: "JENDL-4.0: A New Library for Nuclear Science and Engineering," J. Nucl. Sci. Technol., 48(1), 1-30 (2011)

[9] P.J. Griffin, C.D. Peters, D.W. Vehar, IEEE Trans. on Nucl. Sci., 59 (2012)

[10] S. Simakov et al., "Validation of IRDFF in ${ }^{252} \mathrm{Cf}$ standard and IRDF-2002 reference neutron fields," paper presented at ISRD15 in May 2014 and pending publication in the European Physics Journal 